\title{
From Double-Four-Ring Germanosilicates to New Zeolites: in silico investigation.
}

\author{
Michal Trachta ${ }^{[\mathrm{b}]}$ Ota Bludský, ${ }^{[\mathrm{b}]}$ Jiří Čejka, ${ }^{[\mathrm{c}]}$ Russell E. Morris, ${ }^{[\mathrm{d}]}$ and Petr \\ Nachtigall ${ }^{*[a]}$
}

The majority of zeolites synthesized up to date was prepared via the solvothermal route using organic structure directing agents. Two new zeolites with structural codes $P C R$ and OKO were recently prepared from UTL germanosilicate by removal of D4R connecting dense twodimensional layers [Nature Chem. 2013, 5, 628]. The corresponding experimental protocol - Assembly-Disassembly-Organization-

\section{Introduction}

The use of germanium in the synthesis of zeolites has led to the discovery of a number of new zeolite structures that were not yet synthesized directly in silicate or aluminosilicate forms ${ }^{[1]} \mathrm{A}$ family of zeolite structures containing a double-four ring (D4R) structural units is an important group among these germanosilicates. While Ge-containing zeolites (Ge-zeolites) are very interesting from the structural point of view their applications are problematic due to their lower stability, caused by easily hydrolysed Ge-O bonds. ${ }^{[2]}$ Very recently three different post-synthesis strategies leading to substantial reduction of $\mathrm{Ge}$ content in the framework were proposed: (i) Isomorphous substitution of $\mathrm{Si}$ for $\mathrm{Ge}$ in germanosilicates under strong acidic conditions; it leads to the formation of siliceous zeolite framework (one that has not yet been prepared by direct synthesis) either with $^{[2 a]}$ or without ${ }^{[2 b]}$ mesopore formation. (ii) A selective removal of germaniumcontaining four-ring units, the so-called inverse $\sigma$ transformation route, has been achieved under strong acidic conditions for UTL Ge-zeolite leading to a new COK-14 zeolite (OKO framework type). $^{[3]}$ (iii) A complete removal of Ge-rich D4R units of UTL zeolite leading to siliceous IPC-1P layered material ${ }^{[2 c]}$ that has been further transformed into two new high-silica zeolites IPC-4 and IPC-2 (PCR and OKO framework types, respectively). ${ }^{[4]}$ Clearly, D4R-containing Ge-zeolites offer fascinating possibilities for post-synthesis modifications, ${ }^{[5]}$ leading even to new zeolite structures. Such possibilities were demonstrated for the UTL zeolite in particular. However, they have not been fully explored yet even for this zeolite.

The isomorphous substitution of $\mathrm{Si}$ for $\mathrm{Ge}$ leads to a siliceous framework of the same topology as parent germanosilicate; the inverse $\sigma$ transformation provided just one new high-silica framework. However, each layered material obtained upon D4R dissolution of any particular D4R-zeolite can lead to several new zeolite structures. This strategy has been described recently as Assembly-Disassembly-Organization-Reassembly

(ADOR) procedure. The IPC-1P layered zeolite (obtained from UTL by
Reassembly $(A D O R)$ - is explored in this contribution using in silico investigation. Structure and properties of hypothetical zeolites that could be obtained using ADOR protocol from zeolites with IWW, IWV, IWR, ITR, and ITH topologies are reported based on computational investigation. Total of 19 new structures are presented together with their characteristics.

chemo-selective hydrolysis) was already transformed into two new zeolite framework types. ${ }^{[4]}$ In addition, several other zeolite frameworks were proposed as possible from the IPC-1P precursor upon suitable re-ordering of layered zeolite precursor (Figure 1) ${ }^{[6]}$ It is a great challenge for the field of zeolite synthesis to find suitable inter-layer reassembly conditions that would lead to zeolites with new framework types. This challenge can be generalized (at least) to all zeolites having D4R units as interlayer pillars, such as (in addition to UTL) IWW, IWV, IWR, ITR, and ITH topologies, particularly those rich in germanium. The feasibility of the ADOR procedure for various framework types is evaluated herein based on calculations at the density functional theory level. The results not only predict the structure of yet unknown zeolites but provide structural and energetic characteristics as useful hints in the experimental quest for corresponding new zeolites.

Several million hypothetical zeolites structures have been proposed and their structures are available in various databases. ${ }^{[7]}$ Over 2 million new zeolite structures were generated based on systematic investigation of all 230 possible crystal space groups with various unit cell sizes and $\mathrm{T}$ atom densities using the Monte Carlo procedure zefsall. ${ }^{[7 \mathrm{~b}]}$ About $10 \%$ of these hypothetical zeolites were predicted (based on force field calculations) to be thermodynamically accessible as aluminosilicates. ${ }^{[7 a, 8]}$ These results clearly show the enormous variety of zeolite structures that may exist. However, the

[a] Prof P Nachtigall

Department of Physical and Macromolecular Chemistry, Faculty of Science, Charles University in Prague, Hlavova 2030, 12840 Prague 2. Czech Republic

E-mail: petr.nachtigall@natur.cuni.cz

[b] M. Trachta, Dr. O. Bludský

Institute of Organic Chemistry and Biochemistry, AS ČR,

Flemingovo nám. 2, 16610 Prague, Czech Republic

[c] Prof. J. Čejka

Department of Synthesis and Catalysis, J. Heyrovský Institute of Physical Chemistry Academy of Sciences of Czech Republic, v.v.i., Dolejškova 3, 18223 Praque 8, Czech Republic

[d] Prof. R. E. Morris

EaStCHEM School of Chemistry, University of St Andrews

St Andrews KY16 9ST, UK

Supporting information for this article is available on the WWW under http://www.chemphyschem.org 
synthesis strategy leading to such new zeolites remains unclear. Additional criteria for evaluation of feasibility of the hypothetical zeolite synthesis has been proposed recently, e. g., local interatomic distances ${ }^{[9]}$ or flexibility window over a range of densities. ${ }^{[10]}$

The focus of the present investigation is rather different - only 20 new zeolite structures are presented below based on computational investigation. However, the synthesis of all these zeolites can be attempted using a recently discovered experimental procedure and it is reasonable to believe that some of these zeolites can really be obtained experimentally in the near future. The computational strategy adopted herein closely follows the experimental ADOR protocol depicted in Figure 1: a zeolite with suitable architecture was selected (Assembly); a layered material was simply obtained by a removal D4R units (Disassembly); various interlayer connectivity patterns were considered (Organization); for topologically unique 3D structures the geometries were fully optimized at the density functional level of theory (Reassembly) and basic structural characteristics were evaluated. Due to a moderate number of zeolite structures investigated herein an accurate dispersion-corrected DFT approach is adopted instead of commonly used force fields.

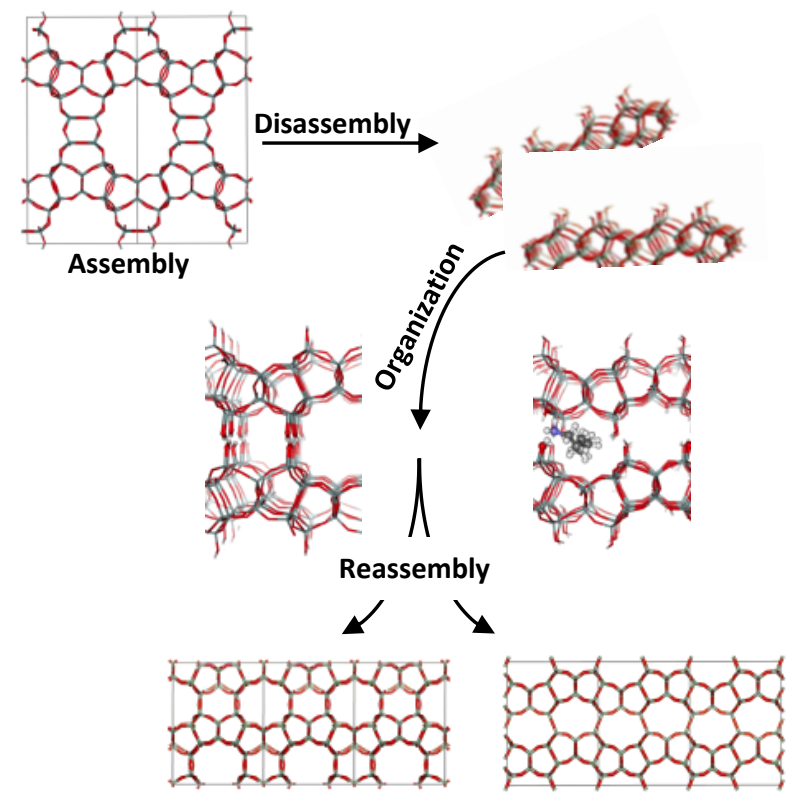

Figure 1. Synthesised zeolites (Assembly) containing D4R units can be transformed into layered material (Disassembly); using specific structuredirecting agent layers may form an ordered inter-layer $\mathrm{H}$-bonded network (Organization) calcination of which (Reassembly) lead to new framework type (shown for UTL zeolite; framework oxygen atoms depicted in red). Topological analysis shows that 20 new unique framework types can be obtained by direct condensation of layers obtained by D4R removal from UTL, IWW, IWV, IWR, ITH, and ITR.

\section{Results and Discussion}

The ADOR protocol is ideal for zeolites where the threedimensional (3D) structure can be chemo-selectively hydrolysed into 2D zeolite layers. The group of zeolites containing Ge-rich D4R pillars connecting 2D layers is particularly suitable for this approach (Fig. 1). Zeolites with the following topologies that fit
Table 1. Relative stabilities, framework densities and channel characteristics of parent and new zeolites.

\begin{tabular}{|c|c|c|c|c|c|}
\hline Zeolite $^{[\mathrm{a}]}$ & $E_{\mathrm{rel}}^{[\mathrm{b}]}$ & Channel architecture & $\mathrm{FD}^{[\mathrm{c}]}$ & $\mathrm{V}_{\text {micro }}{ }^{[\mathrm{d}]}$ & $\mathrm{SA}^{\mathrm{ee}}$ \\
\hline UTL & 12.0 & 2D: $14 \mathrm{R} \times 12 \mathrm{R}$ & 15.1 & 0.265 & 779 \\
\hline$-\mathrm{D} 4 \mathrm{R}(\mathrm{C} 2 / \mathrm{m})$ & 9.1 & 2D: $10 \mathrm{R}$ x 8R & 18.1 & 0.137 & 209 \\
\hline$-\mathrm{D} 4 \mathrm{R}(\mathrm{Pm})$ & 11.7 & $2 \mathrm{D}: 8 \mathrm{R} \times 8 \mathrm{R}$ & 19.0 & 0.049 & 43 \\
\hline$-D 4 R(P 1)$ & 12.5 & 1D: $10 \mathrm{R}$ & 18.7 & 0.059 & 76 \\
\hline \multirow[t]{2}{*}{-D4R(Pm') } & 14.7 & 1D: $8 \mathrm{R}$ & 19.3 & 0.044 & 5 \\
\hline & & $3 D: 12 R+8 R \times 10 R x$ & & & \\
\hline IWW & 11.7 & $10 \mathrm{R}$ & 15.9 & 0.21 & 577 \\
\hline -D4R(Pbam) & 11.0 & $3 D: 12 R+8 R \times 8 R$ & 17.9 & 0.137 & 206 \\
\hline$-D 4 R(C 2 / c)$ & 19.0 & OD & 19.9 & 0.157 & 210 \\
\hline -D4R(Aba2) & 22.3 & OD & 20.7 & 0.133 & 136 \\
\hline IWV & 13.3 & $2 \mathrm{D}: 14 \mathrm{R} \times 12 \mathrm{R}$ & 14.7 & 0.299 & 789 \\
\hline -D4R(Fmmm) & 11.1 & 2D: $10 \mathrm{R}$ x 8R & 18.0 & 0.175 & 134 \\
\hline$-\mathrm{D} 4 \mathrm{R}(\mathrm{Cmm} 2)$ & 16.8 & $2 \mathrm{D}: 8 \mathrm{R} \times 8 \mathrm{R}$ & 18.9 & 0.149 & 61 \\
\hline$-\mathrm{D} 4 \mathrm{R}(\mathrm{C} 2 / \mathrm{m})$ & 21.2 & 1D: 8R & 20.2 & 0.061 & 16 \\
\hline IWR & 12.1 & 3D: $12 \mathrm{R} \times 10 \mathrm{R} \times 10 \mathrm{R}$ & 15.2 & 0.262 & 774 \\
\hline$-\mathrm{D} 4 \mathrm{R}\left(\mathrm{P} 1_{\mathrm{Cmmm}}\right)$ & 11.3 & $3 D: 12 R \times 8 R \times 8 R$ & 17.1 & 0.173 & 423 \\
\hline$-\mathrm{D} 4 \mathrm{R}(\mathrm{C} 2 / \mathrm{m})$ & 11.5 & $2 \mathrm{D}: 8 \mathrm{R} \times 8 \mathrm{R}$ & 17.8 & 0.126 & 222 \\
\hline$-\mathrm{D} 4 \mathrm{R}(\mathrm{Cmmm})$ & 12.3 & $3 D: 12 R \times 8 R \times 8 R$ & 17.1 & 0.175 & 411 \\
\hline -D4R(Fmmm) & 13.3 & 3D: $8 \mathrm{R} \times 8 \mathrm{R} \times 8 \mathrm{R}$ & 18.0 & 0.168 & 376 \\
\hline ITH & 10.4 & $3 \mathrm{D}: 10 \mathrm{R} \times 9 \mathrm{R} \times 10 \mathrm{R}$ & 16.8 & 0.174 & 426 \\
\hline$-\mathrm{D} 4 \mathrm{R}\left(P 1_{A m m 2}\right)$ & 8.9 & 3D: $9 R \times 8 R \times 8 R$ & 19.1 & 0.090 & 73 \\
\hline$-\mathrm{D} 4 \mathrm{R}(\mathrm{Cm})$ & 9.7 & 1D: $8 \mathrm{R}$ & 19.3 & 0.056 & 7 \\
\hline$-\mathrm{D} 4 \mathrm{R}(A m m 2)$ & 9.9 & $3 \mathrm{D}: 9 \mathrm{R} \times 8 \mathrm{R} \times 8 \mathrm{R}$ & 19.0 & 0.094 & 71 \\
\hline$-\mathrm{D} 4 \mathrm{R}\left(\mathrm{Cm} \mathrm{m}^{\prime}\right)$ & 11.2 & OD & 19.5 & 0.130 & 27 \\
\hline ITR & 10.4 & 3D: $10 \mathrm{R} \times 10 \mathrm{R} \times 9 \mathrm{R}$ & 16.8 & 0.176 & 432 \\
\hline$-\mathrm{D} 4 \mathrm{R}(\mathrm{P} 21 / \mathrm{m})$ & 8.3 & 3D: $9 \mathrm{R} \times 8 \mathrm{R} \times 8 \mathrm{R}$ & 19.3 & 0.084 & 23 \\
\hline$-D 4 R(P-1)$ & 8.3 & $3 \mathrm{D}: 9 \mathrm{R} \times 8 \mathrm{R} \times 8 \mathrm{R}$ & 19.4 & 0.069 & 10 \\
\hline$-\mathrm{D} 4 \mathrm{R}(\mathrm{C} 2 / \mathrm{m})$ & 8.4 & 3D: $9 R \times 8 R \times 8 R$ & 19.4 & 0.073 & 8 \\
\hline$-\mathrm{D} 4 \mathrm{R}\left(\mathrm{C} 2 / \mathrm{m}^{\prime}\right)$ & 8.7 & 3D: $9 \mathrm{R} \times 8 \mathrm{R} \times 8 \mathrm{R}$ & 19.4 & 0.083 & 35 \\
\hline
\end{tabular}

[a] D4R-containing "parent" zeolites (in bold) and hypothetical zeolites derived from parent zeolites by in silico ADOR procedure. [b] Relative energies with respect to $\alpha$-quartz in $\mathrm{kJ} \mathrm{mol}^{-1} \mathrm{Si}$. [c] Framework density in Si per $1000 \AA^{3}$.[d] Microporous volume, in $\mathrm{cm}^{3} \mathrm{~g}^{-1}$. [e] Surface area, in $\mathrm{m}^{2} \mathrm{~g}^{-1}$.

this criterion (denoted below "parent" zeolites) were therefore investigated: UTL, IWW, IWV, IWR, ITH, and ITR. It should be noted that ADOR protocol requires the presence of $\mathrm{Ge}$ in $\mathrm{D} 4 \mathrm{R}$ and not all of the parent zeolites investigated herein are at present known in a germanosilicate form. Relative energies and basic characteristics of channel system are summarized in Table 1; additional data, including unit cell parameters, coordination sequences, vertex symbols, zeolite structures (cif format), and corresponding powder XRD patterns are given in Supporting Information. Following the strategy outlined in the Computational Section, a total of 20 new zeolite framework types were identified. Four new zeolite structures were identified for UTL and ITR parent zeolites while, due to symmetry reasons, only three topologically unique zeolite structures were found for the other parent zeolites. The new zeolite framework types listed in Table 1 are classified by their symmetry and their relative energies per $\mathrm{SiO}_{4 / 2}$ tetrahedron $\left(E_{\text {rel }}\right)$ calculated at the vdW-DF2 level of theory are reported with respect to $\alpha$-quartz. The following notation based on the parent zeolite framework type is adopted: L-XYZ$\mathrm{D} 4 \mathrm{R}$ is the 2D layer obtained from $\mathrm{XYZ}$ zeolite (IZA code) upon hydrolysis of D4R units and XYZ-D4R(SYM) denotes a new framework type of $S Y M$ symmetry, obtained from $X Y Z$ parent zeolite by condensation of corresponding layers. Results for IWR- 
$\mathrm{D} 4 \mathrm{R}\left(\mathrm{P} 1_{\mathrm{Cmmm}}\right)$ and for ITH-D4R(P1 $\left.1_{\mathrm{Amm} 2}\right)$ are also included; these zeolites do not represent new topologies (they are identical to zeolites IWR-D4R(Cmmm) and ITH-D4R(Amm2), respectively). However, the lowering of symmetry leads to a significant change (over $1 \mathrm{~kJ} \mathrm{~mol}^{-1} \mathrm{Si}$ ) of relative energy. Only the results for UTLD4R family of zeolites are discussed in detail below while for the other zeolites the details can be found in the Supporting Information and the main text focuses on general features based on theoretical investigation.
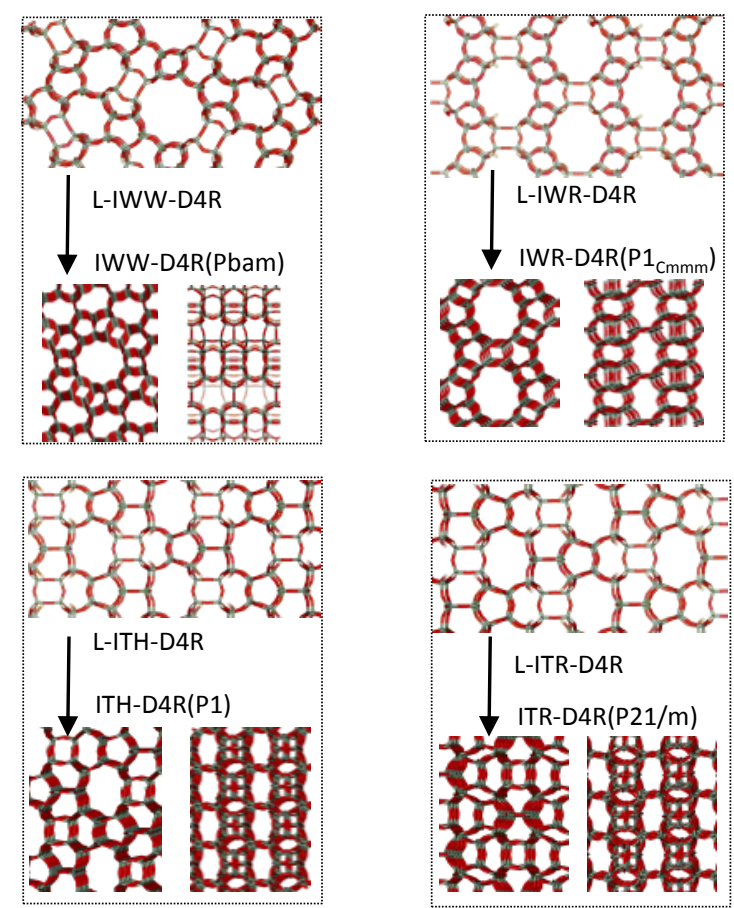

Figure 2. Layered zeolite materials derived from IWW, IWR, ITH, and ITR zeolites (upper parts; viewed along the normal of $2 \mathrm{D}$ dense layers) and energetically the most favourable 3D zeolites obtained by an ordered condensation of layers (viewed along the channels).

The UTL-D4R family of zeolites is discussed first since the UTL-D4R(C2/m) zeolite was already obtained experimentally following the ADOR protocol (zeolite IPC- $4^{[4]}$ - PCR framework type assigned recently by International Zeolite Association). ${ }^{[11]}$ Removal of the D4Rs from the parent UTL structure leads to the layered material denoted L-UTL-D4R (this has been already achieved experimentally ${ }^{[2]}$ and the material obtained has been denoted IPC-1P). In agreement with previous work ${ }^{[4]}$ the analysis based on objective function surface described in the Computational Section led to four topologically unique zeolites (for coordination sequences and vertex symbols see Table S2). Among them, the zeolite denoted UTL-D4R $(\mathrm{C} 2 / \mathrm{m})$, corresponding to PCR framework type, is energetically the most stable. This zeolite is obtained from L-UTL-D4R layers by the direct condensation of silanols connected in parent UTL zeolite via D4R units. The removal of D4R units separating L-UTL-D4R layers in the parent UTL zeolite led to the reduction of channel dimensions from $14 R \times 12 R$ in parent UTL to $10 R \times 8 R$ in UTL-D4R(C2/m) while the orientation of channels remained unaffected by the UTL $\rightarrow$ UTL-D4R(C2/m) transformation. The micropore volume 0.137 $\mathrm{cm}^{3} \mathrm{~g}^{-1}$ and surface area $209 \mathrm{~m}^{2} \mathrm{~g}^{-1}$ calculated using the geometric approach are in good agreement with experimental values $\left(0.106 \mathrm{~cm}^{3} \mathrm{~g}^{-1} \text { and } 236 \mathrm{~m}^{2} \mathrm{~g}^{-1} \text {, respectively }\right)^{[4]}$ obtained from nitrogen-adsorption experiments at $77 \mathrm{~K}$ (BET analysis). Note that similar $30 \%$ overestimation of micropore volume was also found for parent UTL zeolite, comparing geometrical approach and BET analysis $\left(0.265\right.$ and $0.190 \mathrm{~cm}^{3} \mathrm{~g}^{-1}$, respectively). Such consistency justifies the use of geometrical approach adopted here for characterization of zeolites micropores. The structure of UTL-D4R $(\mathrm{C} 2 / \mathrm{m})$ zeolite obtained computationally at the DFT level is very close to the experimental structure (see Ref. ${ }^{[4]}$ for details). Note also that the UTL $\rightarrow$ UTL-D4R(C2/m) transformation leads to an increase of the framework density and a decrease of relative energy with respect to $\alpha$-quartz (by $2.5 \mathrm{~kJ}$ $\mathrm{mol}^{-1} \mathrm{Si}$ at least). This new zeolite stays on the energy versus density line defined by existing D4R-containing zeolites (Figure S1 in Supporting Information). The other three zeolites of the UTL-D4R family are characterized by significantly smaller pore volume and surface area, higher framework density, and higher relative energy than UTL-D4R(C2/m) one. A large energy difference between individual zeolites of UTL-D4R family sets an extra challenge for experimental investigators to find such conditions that could lead selectively to a different connectivity than those in UTL-D4R(C2/m) zeolite.

Zeolite IWV has similar structural features as UTL - compact IWV layers are connected via D4R pillars forming a 2D channel system consisting of $14 R$ and $12 R$ windows. The analysis of IWVD4R family of zeolites shows a marked similarity to UTL-D4R family of zeolites. The IWV-D4R(Fmmm) zeolite has $8 R$ channels interconnected by $10 R$ windows, similar to UTL-D4R $(C 2 / m)$. This new IWV-D4R(Fmmm) zeolite is characterized with micropore volume of $0.175 \mathrm{~cm}^{3} \mathrm{~g}^{-1}$ and surface area of $134 \mathrm{~m}^{2} \mathrm{~g}^{-1}$.

UTL and IWV framework types have 2D channel systems, thus, their layered analogues obtained upon removal of D4Rs do not have any $8 \mathrm{R}$ or large channel through the lamellae. On the contrary, IWW, IWR, ITH, and ITR framework types possess a 3D channel system, therefore, the corresponding $2 \mathrm{D}$ layered zeolites have micropores through the lamella (Figure 2). Energetically the most favorable zeolites obtained from IWW, IWR, ITH, and ITR are also depicted in Figure 2. Channels through L-IWW-D4R, LIWR-D4R, and L-ITH-D4R lamellas $(12 R, 12 R$, and 9R, respectively) are preserved in IWW-D4R(Pbam), IWR-D4R(P1Cmmm), and ITH-D4R(P1) zeolites. On the contrary, the size of the channel is significantly reduced upon ITR $\rightarrow$ ITR-D4R(P21/m) transformation.

All new zeolite structures reported in Table 1 appear to be thermodynamically accessible as aluminosilicates (they are within $30 \mathrm{~kJ} \mathrm{~mol}^{-1} \mathrm{Si}$ of $\alpha$-quartz, Ref. ${ }^{[8]}$ ). The energy versus density (defined as the number of $\mathrm{Si}$ atoms per $1000 \AA^{3}$ ) plot for parent and new zeolites is shown in Figure S1 in Supporting Information. A large energy difference between individual framework types increases chances that ADOR procedure leads to ordered crystalline material. Large energy differences $\left(E_{\text {rel }}\right)$ were found for zeolites in IWW-D4R and IWV-D4R families (8 and $5.7 \mathrm{~kJ} \mathrm{~mol}^{-1} \mathrm{Si}$, respectively). The IWW-D4R(Pbam) zeolite appears to be a particularly suitable candidate to be obtained by ADOR procedure experimentally; it has $12 R$ and $8 R$ channels along the L-IWW$\mathrm{D} 4 \mathrm{R}$ normal that are interconnected by narrow $8 \mathrm{R}$ channel formed in between individual layers (Figure S2). Note that this channel does not result from 10R channel present in parent IWW zeolite (the size of this channel is reduced to $6 \mathrm{R}$ ).

While there is always one energetically strongly favored framework type in UTL-D4R, IWV-D4R, and IWW-D4R families, the situation is distinctly different in IWR-D4R, ITH-D4R, and ITRD4R families, where several framework types have similar energies (in ITR-D4R family in particular, Figure S1). On one hand, it gives a chance to experimentally prepare more than one 
new framework type out of one layered material, on the other hand, it is likely that condensation of L-IWR-D4R, L-ITH-D4R, and L-ITR-D4R layered materials could lead to an irregular 3D materials where adjacent layers are connected in different ways. An extra demand on the proper choice of interlayer reassembly conditions that would lead to regular structure can be foreseen. Note that L-ITH-D4R and L-ITR-D4R are identical; the difference between parent ITH and ITR zeolite is only in the relative orientation of neighboring 2D layers and their connection by $\mathrm{D} 4 \mathrm{R}$ pillars.

Since all new zeolite structures reported herein are obtained by in silico condensation of layers obtained from parent zeolite upon removal of D4R units their surface areas (SA) and micropore volumes $\left(\mathrm{V}_{\text {micro }}\right)$ are smaller than those in parent zeolites. Among 20 zeolites proposed only 6 have surface area larger than $200 \mathrm{~m}^{2} \mathrm{~g}^{-1}$ although this is not necessarily a negative feature given the renewed interest in small pore zeolites. ${ }^{[12]}$ The new zeolites of the IWR-D4R family are perhaps the most interesting - all 3 of them have interesting channel architecture and $\mathrm{SA}>200 \mathrm{~m}^{2} \mathrm{~g}^{-1}$; in addition, all these new zeolites are energetically no more than $13 \mathrm{~kJ} \mathrm{~mol}^{-1} \mathrm{Si}$ above $\alpha$-quartz and due to their distinct channel architecture chances are that several of them could be obtained experimentally with perhaps the proper choice of specific SDA. On the contrary, all zeolites of ITR-D4R and ITH-D4R families have only small surface area and small $V_{\text {micro, }}$ thus, they do not appear to be suitable for applications.

Structures with number of unique $T$ atoms not exceeding eight (8 out of 20 , Table S2) were compared with previously reported hypothetical zeolite structure in the Atlas of Prospective zeolite structures. ${ }^{[13]}$ A match was found for five structures - IWRD4R(Cmmm), IWR-D4R(Fmmm), IWR-D4R(C2/m), ITH-D4R(Cm), and IWV-D4R(Cmm2), they were all found in DEEM DATABASE (only three out of these eight zeolites were not found in this database). Link to matching structures can be found in Supporting information.

\section{Conclusion}

In summary, the structure and thermodynamic stability of hypothetical new zeolites obtained by the ADOR protocol were evaluated theoretically at the vdW-DF2 level of theory. A total of 20 new zeolite topologies were found. One of them, UTL$\mathrm{D} 4 \mathrm{R}(\mathrm{C} 2 / \mathrm{m})$ has been already obtained by ADOR protocol. Among the remaining 19 (at this moment) hypothetical zeolites, the IWW-D4R(Pbam) and IWV-D4R(Fmmm) are the most promising candidates for experimental research based on their relative thermodynamic stability. Zeolites of the IWR-D4R family have the largest surface area and $V_{\text {micro }}$ and complex $3 D$ channel system. Due to the relatively small differences in their thermodynamic stability the synthesis of these zeolites may require a use of specific structure-directing agent molecules that can be designed, e.g., with the help of ZEBEDDE computational method. $^{[14]}$

\section{Computational Section}

The structures of two-dimensional layers were obtained from IWW, IWV, UTL, ITH, IWR, and ITR zeolites simply by removal of the D4R units. The structural parameters of the parent zeolites were taken from the database of zeolite structures (IZA) ${ }^{[11]}$ As demonstrated for UTL a novel 3D zeolite framework PCR can be synthesized by topotactic solid-state condensation of the $2 \mathrm{D}$ layered precursors IPC-1P. ${ }^{[4]}$ The reassembly of the $2 \mathrm{D}$ zeolite frameworks can be, however, achieved in many ways depending on the distribution of the surface silanols originated from the dissolved D4R units. To explore possible arrangements of the layers we have adopted a two-step procedure consisting of (i) minimizing the $\mathrm{O}-\mathrm{O}$ distance between overlapping silanol groups that

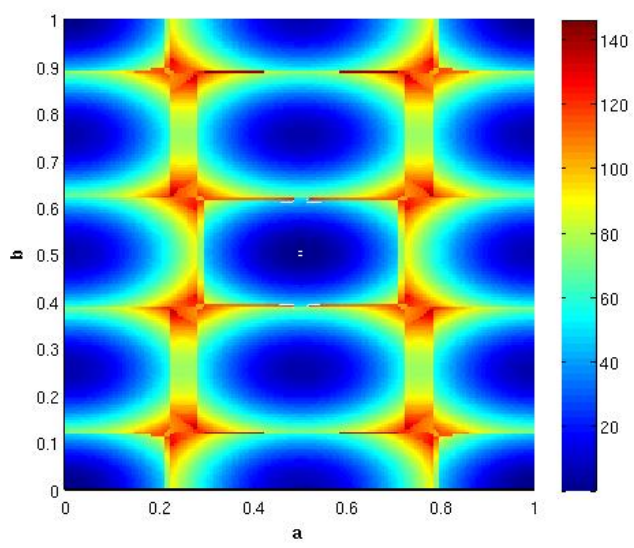

undergo the topotactic condensation and (ii) optimizing the structural parameters of the condensed 3D zeolite framework by periodic DFT calculations. In the first step, the $2 \mathrm{D}$ zeolite frameworks were kept fixed at the

Figure 3. Modelling the organization phase of ADOR procedure. The objective function $\mathrm{X}$ (in $\AA^{2}, a$ and $b$ in fractional coordinates) minimizing the interlamellar distances of silanol oxygens for various relative positions of two L-ITH-D4R lamellas. With respect to periodicity, there are 8 distinct minima and thus 8 possible zeolite structures (just 3 of them have unique topologies).

geometries found in the parent materials including oxygens of silanol groups. The $\mathrm{O}-\mathrm{O}$ distances of overlapping silanols were minimized in the least-squares sense by optimizing the relative positions of the rigid layers. Only translations of layers were considered during optimization process. The objective function $X$ is defined as the sum of squares of the shortest $\mathrm{O}-\mathrm{O}$ distances between silanols of neighboring layers; an example for $2 \mathrm{D}$ layers derived from the ITH zeolite is depicted in Figure 3. The function $x$ depends on a relative position of layers given in fractional coordinates with respect to the original ITH cell. The local minima on the objective function surface correspond to 8 structures with a favorable arrangement of surface silanols for the topotactic condensation. Our algorithm generating starting geometries for $a b$ initio structural optimizations is based on the assumption that the distances between silanol groups correlate with the framework deformation energy required for creating the interlayer Si-OSi bonds. The proposed procedure guarantees neither finding a complete set of topological connectivities between zeolite layers nor finding all local minima on the corresponding potential energy surface. However, our starting structures should be reasonable if the geometries of the $2 \mathrm{D}$ layer frameworks do not change too much when going from a parent zeolite to the $3 \mathrm{D}$ material synthesized by solid-state condensation.

Only topologically unique structures were considered in the second step. Topology analysis (coordination sequences and vertex symbols) was carried out using a home-made program written in MATLAB. The full geometry optimizations including the unit cell size and shape were carried out for selected $3 \mathrm{D}$ structures at the $a b$ initio (DFT) level of theory. The periodic DFT calculations were performed with the VASP program suite ${ }^{[15]}$ using PerdewBurke-Erzenhof (PBE) density functional ${ }^{[16]}$ and the projector augmented wave (PAW) approximation. ${ }^{[17]}$ The geometry optimizations were carried out using the standard PAW/PBE pseudopotentials with ENMAX values of 245, 400 and 250 $\mathrm{eV}$ for silicon, oxygen and hydrogen, respectively. The kinetic energy cutoff of $800 \mathrm{eV}$ was employed to limit the error in diagonal components of the stress tensor during cell shape and volume relaxations. In the geometry optimizations the convergence condition of $0.01 \mathrm{eV} \AA^{-1}$ was utilized for the ionic relaxation. The total energies for the optimized structures were obtained using the vdWDF2 non-local exchange-correlation functional ${ }^{[18]}$ and soft PAW/PBE pseudopotentials. Use of the non-local functional appeared to be essential for correct assessment of relative energies with respect to $\alpha$-quartz. The Brillouinzone sampling was restricted to the $\Gamma$-point only; $\Gamma$-point sampling should be sufficient for periodic calculations with unit cell sizes in the range of $1500-8000$ $\AA^{3}$. ${ }^{[19]}$

Geometric approach for microporous volume and for surface area was adopted; Connolly surface area ${ }^{[20]} \mathrm{SA}$ and solvent micorporous volume $\mathrm{V}_{\text {micro }}$ (using $\mathrm{Ar}$ probe with diameter of $1.84 \AA$ ) were evaluated. 


\section{Acknowledgements}

Authors acknowledge the financial support from the Grant Agency of the Czech Republic (Center of Excellence - P106/12/G015). R.E.M. thanks the Royal Society for provision of an industry fellowship and the E.P.S.R.C. for funding (EP/K025112/1).

\section{Keywords: zeolites $\bullet$ structure $\bullet$ synthesis $\bullet$ in silico $\bullet$ DFT}

[1] [1] J. X. Jiang, J. H. Yu, A. Corma, Angew. Chem. Int. Ed. 2010, 49, 3120-3145.

[2] a) L. Burel, N. Kasian, A. Tuel, Angew. Chem. Int. Ed. 2014, 53, 1360-1363; b) H. Xu, J.-G. Jiang, B. Yang, L. Zhang, M. He, P. Wu, Angew. Chem. Int. Ed. 2014, 53, 1355-1359; c) W. J. Roth, O. V. Shvets, M. Shamzhy, P. Chlubna, M. Kubu, P. Nachtigall, J. Cejka, J. Am. Chem. Soc. 2011, 133, 6130-6133; d) W. J. Roth, P. Nachtigall, R. E. Morris, J. Čejka, Chem. Rev. 2014, 114, 48074837.

[3] E. Verheyen, L. Joos, K. Van Havenbergh, E. Breynaert, N. Kasian, E. Gobechiya, K. Houthoofd, C. Martineau, M. Hinterstein, F. Taulelle, V. Van Speybroeck, M. Waroquier, S. Bals, G. Van Tendeloo, C. E. A. Kirschhock, J. A. Martens, Nat. Mater. 2012, 11, 1059-1064.

[4] W. J. Roth, P. Nachtigall, R. E. Morris, P. S. Wheatley, V. R. Seymour, S. E. Ashbrook, P. Chlubna, L. Grajciar, M. Polozij, A. Zukal, O. Shvets, J. Cejka, Nat. Chem. 2013, 5, 628-633.

[5] P. Chlubna, W. J. Roth, H. F. Greer, W. Z. Zhou, O. Shvets, A Zukal, J. Cejka, R. E. Morris, Chem. Mater. 2013, 25, 542-547.

[6] L. Grajciar, O. Bludsky, W. J. Roth, P. Nachtigall, Catal. Today 2013, 204, 15-21.

[7] a) M. W. Deem, R. Pophale, P. A. Cheeseman, D. J. Earl, J. Phys. Chem. C 2009, 113, 21353-21360; b) M. W. Deem, J. M. Newsam, Nature 1989, 342, 260-262; c) A. Le Bail, J. Appl. Crystallogr. 2005, 38, 389-395; d) M. O'Keeffe, M. A. Peskov, S. J. Ramsden, O. M. Yaghi, Acc. Chem. Res. 2008, 41, 1782-1789; e) M. M. J. Treacy, I. Rivin, E. Balkovsky, K. H. Randall, M. D. Foster, Microporous Mesoporous Mater. 2004, 74, 121-132; f) R. Pophale, P. A. Cheeseman, M. W. Deem, Phys. Chem. Chem. Phys. 2011, 13, 12407-12412; g) J. M. Thomas, J. Klinowski, Angew. Chem. Int. Ed. 2007, 46, 7160-7163; h) C. J. Dawson, M. A. B. Pope, M. O'Keefie, M. M. J. Treacy, Chem. Mater. 2013, 25, 3816-3821.

[8] M. D. Foster, A. Simperler, R. G. Bell, O. D. Friedrichs, F. A. A. Paz, J. Klinowski, Nat. Mater. 2004, 3, 234-238.

[9] Y. Li, J. H. Yu, R. R. Xu, Angew. Chem. Int. Ed. 2013, 52, 16731677.

[10] A. Sartbaeva, S. A. Wells, M. M. J. Treacy, M. F. Thorpe, Nat. Mater. 2006, 5, 962-965.

[11] C. Baerlocher, L. B. McCusker, Database of Zeolite Structures: http://www.iza-structure.org/databases/.

[12] a) M. Hernandez-Rodriguez, J. L. Jorda, F. Rey, A. Corma, J. Am. Chem. Soc. 2012, 134, 13232-13235; b) M. Moliner, C. Martinez, A. Corma, Chem. Mater. 2014, 26, 246-258.

[13] M. D. Foster, M. M. J. Treacy, Database of Hypothetical Zeolite Structures: http://www.hypotheticalzeolites.net.

[14] D. W. Lewis, D. J. Willock, C. R. A. Catlow, J. M. Thomas, G. J. Hutchings, Nature 1996, 382, 604-606.

[15] G. Kresse, J. Hafner, Phys. Rev. B 1993, 48, 13115.

[16] J. P. Perdew, K. Burke, M. Ernzerhof, Phys. Rev. Lett. 1996, 77, 3865.

[17] P. E. Blochl, Phys. Rev. B 1994, 50, 17953.

[18] K. Lee, E. D. Murray, L. Z. Kong, B. I. Lundqvist, D. C. Langreth, Phys Rev B 2010, 82, 081101.

[19] M. Rubeš, O. Bludský, ChemPhysChem 2009, 10, 1868-1873.

[20] M. L. Connolly, J. Appl. Crystallogr. 1983, 16, 548-558.

Received: ((will be filled in by the editorial staff))

Published online: ((will be filled in by the editorial staff)) 


\section{Entry for the Table of Contents}

\section{ARTICLES}

Topological analysis shows that 19 new zeolite structures can be obtained by ADOR (AssemblyDisassembly-OrganizationReassembly) procedure from UTL, IWW, IWV, IWR, ITH, and ITR zeolites. Structural and thermodynamic data for those 20 new zeolites are provided based on density functional level of theory. Results reported should serve as a useful hint in the experimental quest for new zeolite structures.

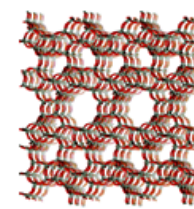

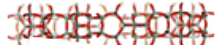

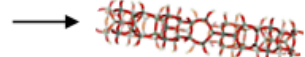

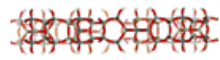

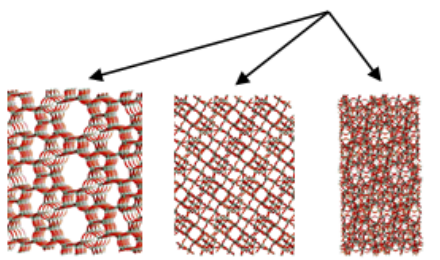

Michal Trachta, Ota Bludský, Jiří Čejka, Russell E. Morris, and Petr Nachtigall, *

Page No. - Page No.

Transformation of Double-FourRing-Containing Zeolites into Structurally New Zeolites. 